\title{
QUASI-PROBABILITY DISTRIBUTION OF NONCLASSICAL STATES IN INTERACTING FOCK SPACE
}

\author{
P. K. DAS and ARPITA GHOSH \\ Physics and Applied Mathematics Unit \\ Indian Statistical Institute \\ 203, B. T. Road, Kolkata-700108, India \\ E-mail:daspk@isical.ac.in,arpitag_r@isical.ac.in
}

\begin{abstract}
In this paper we study approximate quasi-probability distribution functions of nonclassical states such as incoherent states, Kerr states, squeezed states and $k$-photon coherent states in interacting Fock space.
\end{abstract}

1. Introduction. Sometimes it is useful to describe the state of the field in terms of coherent states rather than with photon number states $[3,5,6,7]$. This presents some difficulties as the coherent states are not orthogonal and are overcomplete. However, this overcompleteness allows us to obtain a useful diagonal expansion of the density operator in terms of complex matrix elements $P(\alpha)$. This representation is called a quasi-probability distribution function as it is not positive definite. Quasi-probability function is applied in the evaluation of correlation functions of the electromagnetic field. Dynamics of quasiprobability distribution function can under appropriate conditions be expressed in the form of a Fokker-Planck equation. As the position and momentum cannot be defined simultaneously with an infinite precision, the description of the quantum mechanical state in phase space is not unique. Hence there are a family of quasi-probabilities of which the Glauber-Sudarshan $P$, Husimi $Q$ and Wigner functions are widely used.

Phase space methods have been widely used in quantum mechanics and in quantum optics. Within the framework of the phase space formalism, a state of a quantum mechanical system can be completely described with the help of quasi-probability density distributions. From an operational point of view $Q$ function plays a very important role

2000 Mathematics Subject Classification: Primary 81S05; Secondary 47N50, 81S25.

Key words and phrases: interacting Fock space, quasi-probability distribution, Kerr state, squeezed state, $k$-photon coherent state.

The paper is in final form and no version of it will be published elsewhere. 
in quantum mechanics and can be associated with a simultaneous measurement of two conjugated observables.

In this paper we shall discuss the behavior of $Q$ functions for incoherent states, Kerr states, squeezed states and $k$-photon coherent states for large photon numbers in the interacting Fock space [8]. In this method the lengthy and complicated infinite sums of the exact result reduce to a simpler analytic expression. Thus we gain a deeper insight into the physics and mathematics of the quasi-probability function and the importance of the quasi-probability approach is visualized.

The paper is organized as follows. In section 2, we describe the basic facts of interacting Fock space which we will need throughout the paper. In section 3, we discuss quasiprobability functions for incoherent states, Kerr states, squeezed states and $k$-photon coherent states in details. In section 4 , we give a conclusion.

2. Basic concepts. Here we discuss some basic concepts which will be utilized throughout the paper.

As a vector space one mode interacting Fock space $\Gamma(\mathbb{C})$ is defined by

$$
\Gamma(\mathbb{C})=\bigoplus_{n=0}^{\infty} \mathbb{C}|n\rangle .
$$

where $\mathbb{C}|n\rangle$ is called the $n$-particle subspace. The different $n$-particle subspaces are orthogonal, that is, the sum in (1) is orthogonal. The norm of the vector $|n\rangle$ is given by

$$
\langle n \mid n\rangle=\lambda_{n}
$$

where $\left\{\lambda_{n}\right\} \geq 0$ and if for some $n$ we have $\left\{\lambda_{n}\right\}=0$, then $\left\{\lambda_{m}\right\}=0$ for all $m \geq n$. The norm introduced in $(2)$ makes $\Gamma(\mathbb{C})$ a Hilbert space.

An arbitrary vector $f$ in $\Gamma(\mathbb{C})$ is given by

$$
f \equiv c_{0}|0\rangle+c_{1}|1\rangle+c_{2}|2\rangle+\ldots+c_{n}|n\rangle+\ldots
$$

for any $n \in \mathbb{N}$ with $\|f\|=\left(\sum_{n=0}^{\infty}\left|c_{n}\right|^{2} \lambda_{n}\right)^{1 / 2}<\infty$.

We now consider the following actions on $\Gamma(\mathbb{C})$ :

$$
\begin{aligned}
a^{*}|n\rangle & =|n+1\rangle, \\
a|n+1\rangle & =\frac{\lambda_{n+1}}{\lambda_{n}}|n\rangle, \\
a|0\rangle & =0 .
\end{aligned}
$$

$a^{*}$ is called the creation operator and its adjoint $a$ is called the annihilation operator. To define the annihilation operator we have taken the convention $0 / 0=0$.

We observe that

$$
\langle n \mid n\rangle=\left\langle a^{*}(n-1), n\right\rangle=\langle(n-1), a n\rangle=\frac{\lambda_{n}}{\lambda_{n-1}}\langle n-1, n-1\rangle=\ldots
$$

and

$$
\||n\rangle \|^{2}=\frac{\lambda_{n}}{\lambda_{n-1}} \cdot \frac{\lambda_{n-1}}{\lambda_{n-2}} \ldots \frac{\lambda_{1}}{\lambda_{0}}\langle 0 \mid 0\rangle=\lambda_{n} .
$$

Throughout the paper we assume the condition $\lambda_{0}=1$. 
The commutation relation takes the form

$$
\left[a, a^{*}\right]=\frac{\lambda_{N+1}}{\lambda_{N}}-\frac{\lambda_{N}}{\lambda_{N-1}}
$$

where $N$ is the number operator defined by $N|n\rangle=n|n\rangle$.

In a recent paper [4] we have proved that the set $\left\{\frac{|n\rangle}{\sqrt{\lambda_{n}}}, n=0,1,2,3, \ldots\right\}$ forms a complete orthonormal set and the solution of the following eigenvalue equation

$$
a f_{\alpha}=\alpha f_{\alpha}
$$

is given by

$$
f_{\alpha}=\psi\left(|\alpha|^{2}\right)^{-1 / 2} \sum_{n=0}^{\infty} \frac{\alpha^{n}}{\lambda_{n}}|n\rangle
$$

where $\psi\left(|\alpha|^{2}\right)=\sum_{n=0}^{\infty}|\alpha|^{2 n} / \lambda_{n}$. We call $f_{\alpha}$ a coherent vector in $\Gamma(\mathbb{C})$.

Now, we observe that

$$
a a^{*}=\frac{\lambda_{N+1}}{\lambda_{N}}, \quad a^{*} a=\frac{\lambda_{N}}{\lambda_{N-1}} .
$$

We further observe that $\left(\frac{\lambda_{N+1}}{\lambda_{N}}-\frac{\lambda_{N}}{\lambda_{N-1}}\right)$ commutes with both $a^{*} a$ and $a a^{*}$.

3. Quasi-probability distribution. $Q$ function is defined as the diagonal matrix element of the density operator in a pure coherent state

$$
Q\left(f_{\alpha}\right)=\frac{\left\langle f_{\alpha}|\rho| f_{\alpha}\right\rangle}{\pi} .
$$

3.1. Incoherent state. For incoherent state

$$
\rho=\sum_{n=0}^{\infty} p_{n}\left|\frac{n}{\sqrt{\lambda_{n}}}\right\rangle\left\langle\frac{n}{\sqrt{\lambda_{n}}}\right|
$$

with $p_{n} \geq 0, \sum p_{n}=1$.

Now we have

$$
\begin{aligned}
Q\left(f_{\alpha}\right) & =\frac{1}{\pi}\left\langle f_{\alpha}|\rho| f_{\alpha}\right\rangle \\
& =\frac{1}{\pi}\left\langle f_{\alpha}\left|\sum_{n=0}^{\infty} p_{n}\right| \frac{n}{\sqrt{\lambda_{n}}}\right\rangle\left\langle\frac{n}{\sqrt{\lambda_{n}}} \mid f_{\alpha}\right\rangle \\
& =\frac{1}{\pi} \sum_{n=0}^{\infty} p_{n}\left|\left\langle f_{\alpha} \mid \frac{n}{\sqrt{\lambda_{n}}}\right\rangle\right|^{2} \\
& =\frac{1}{\pi} \sum_{n} p_{n}\left|\left\{\psi\left(|\alpha|^{2}\right)\right\}^{-1 / 2} \sum_{m=0}^{\infty} \frac{|\alpha|^{m}}{\lambda_{m}} e^{-i m \theta_{1}}\left\langle m \mid \frac{n}{\sqrt{\lambda_{n}}}\right\rangle\right|^{2}
\end{aligned}
$$




$$
\begin{aligned}
& =\frac{1}{\pi} \sum_{n} p_{n}\left|\left\{\psi\left(|\alpha|^{2}\right)\right\}^{-1 / 2} \frac{|\alpha|^{n}}{\lambda_{n}} e^{-i n \theta_{1}} \lambda_{n} \frac{1}{\sqrt{\lambda_{n}}}\right|^{2} \\
& =\frac{1}{\pi} \sum_{n} p_{n}\left|\left\{\psi\left(|\alpha|^{2}\right)\right\}^{-1 / 2} \frac{|\alpha|^{n}}{\sqrt{\lambda_{n}}} e^{-i n \theta_{1}}\right|^{2} \\
& =\frac{1}{\pi}\left\{\psi\left(|\alpha|^{2}\right)\right\}^{-1} \sum_{n} p_{n} \frac{|\alpha|^{2 n}}{\lambda_{n}} .
\end{aligned}
$$

For $\lambda_{n} \sim n$ ! we get

$$
Q\left(f_{\alpha}\right) \sim \frac{1}{\pi}\left\{\psi\left(|\alpha|^{2}\right)\right\}^{-1} \sum_{n} p_{n} \frac{|\alpha|^{2 n}}{n !}
$$

and with $\left[\psi\left(|\alpha|^{2}\right)\right]^{-1} \sim\left(\sum_{n=0}^{\infty} \frac{|\alpha|^{2 n}}{n !}\right)^{-1}=e^{-|\alpha|^{2}}$ we arrive at

$$
Q\left(f_{\alpha}\right) \sim \frac{1}{\pi} e^{-|\alpha|^{2}} \sum_{n} p_{n} \frac{|\alpha|^{2 n}}{n !} .
$$

3.2. Kerr state. For Kerr state

$$
\left|\phi_{\beta}^{k}\right\rangle=\sum_{n=0}^{\infty}\left[\left\{\psi\left(|\beta|^{2}\right)\right\}^{-1 / 2} \frac{\beta^{n}}{\lambda_{n}} e^{\frac{i}{2} \gamma \frac{\lambda_{n}}{\lambda_{n}-1}\left(\frac{\lambda_{n}}{\lambda_{n}-1}-1\right)}\right]|n\rangle
$$

we have

$$
\begin{aligned}
Q\left(f_{\alpha}\right)= & \frac{1}{\pi}\left|\left\langle f_{\alpha} \mid \phi_{\beta}^{k}\right\rangle\right|^{2} \\
= & \frac{1}{\pi} \mid\left\{\psi\left(|\alpha|^{2}\right)\right\}^{-1 / 2} \sum_{m=0}^{\infty} \frac{|\alpha|^{m}}{\lambda_{m}} e^{-i m \theta_{1}}\langle m|\left\{\psi\left(|\beta|^{2}\right)\right\}^{-1 / 2} \\
& \left.\cdot \sum_{n=0}^{\infty} \frac{|\beta|^{n}}{\lambda_{n}} e^{i n \theta_{2}} e^{\frac{i \gamma}{2} \frac{\lambda_{n}}{\lambda_{n-1}}\left(\frac{\lambda_{n}}{\lambda_{n-1}}-1\right)}|n\rangle\right|^{2} \\
= & \frac{1}{\pi} \mid\left\{\psi\left(|\alpha|^{2}\right)\right\}^{-1 / 2}\left\{\psi\left(|\beta|^{2}\right)\right\}^{-1 / 2} \sum_{m, n=0}^{\infty} \frac{|\alpha|^{m}|\beta|^{n}}{\lambda_{m} \lambda_{n}} e^{-i m \theta_{1}} e^{i n \theta_{2}} \\
& \left.\cdot e^{\frac{i \gamma}{2} \frac{\lambda_{n}}{\lambda_{n-1}}\left(\frac{\lambda_{n}}{\lambda_{n-1}}-1\right)}\langle m \mid n\rangle\right|^{2} \sum_{n=0}^{\infty} \frac{|\alpha|^{n}|\beta|^{n}}{\lambda_{n}^{2}} e^{i n\left(\theta_{2}-\theta_{1}\right)} \\
= & \frac{1}{\pi}\left|\left\{\psi\left(|\alpha|^{2}\right)\right\}^{-1 / 2}\left\{\psi\left(|\beta|^{2}\right)\right\}^{-1 / 2} \sum_{n=0}{ }^{\frac{i \gamma}{2} \frac{\lambda_{n}}{\lambda_{n-1}}\left(\frac{\lambda_{n}}{\lambda_{n-1}}-1\right)} \lambda_{n}\right|^{2} .
\end{aligned}
$$

For $\lambda_{n} \sim n$ ! we get

$$
Q\left(f_{\alpha}\right) \sim \frac{1}{\pi}\left|e^{-|\alpha|^{2} / 2} e^{-|\beta|^{2} / 2} \sum_{n=0}^{\infty} \frac{|\alpha|^{n}|\beta|^{n}}{n !} e^{i n\left(\theta_{2}-\theta_{1}\right)} e^{\frac{i \gamma}{2}\left(n^{2}-n\right)}\right|^{2} .
$$

If we allow to take $|\alpha|=|\beta|$ we arrive at

$$
Q\left(f_{\alpha}\right) \sim \frac{1}{\pi}\left|\sum_{n=0}^{\infty} \frac{|\alpha|^{2 n}}{n !} e^{-|\alpha|^{2}} e^{i n\left(\theta_{2}-\theta_{1}\right)} e^{\frac{i \gamma}{2}\left(n^{2}-n\right)}\right|^{2} .
$$


Using Gaussian approximation to a Poisson distribution we get

$$
\begin{aligned}
Q\left(f_{\alpha}\right) & \sim \frac{1}{\pi}\left|\sum_{n=0}^{\infty}\left(2 \pi|\alpha|^{2}\right)^{-1 / 2} e^{-\frac{(n-|\alpha|)^{2}}{2|\alpha|^{2}}} e^{i n\left(\theta_{2}-\theta_{1}\right)} e^{\frac{i \gamma}{2}\left(n^{2}-n\right)}\right|^{2} \\
& =\frac{1}{2 \pi^{2}|\alpha|^{2}}\left|\sum_{n=0}^{\infty} e^{-(n A-B)^{2}} e^{-1 / 2} e^{B^{2}}\right|^{2} \\
& \sim \frac{1}{2 \pi^{2}|\alpha|^{2}}\left|\frac{1}{A} \frac{\sqrt{\pi}}{2} e^{-1 / 2} e^{B^{2}}\right|^{2} \\
& =\frac{1}{8 \pi|\alpha|^{2}} \frac{1}{e}\left|\frac{1}{A} e^{B^{2}}\right|^{2}
\end{aligned}
$$

where

$$
A=\frac{\left(\frac{1}{|\alpha|^{2}}-i \gamma\right)^{1 / 2}}{\sqrt{2}}, \quad B=\frac{\frac{1}{|\alpha|}+i\left(\theta_{2}-\theta_{1}-\frac{\gamma}{2}\right)}{\sqrt{2}\left(\frac{1}{|\alpha|^{2}}-i \gamma\right)^{1 / 2}} .
$$

Wilson-Gordon et al. [9] determined the range of parameters for which the Kerr states are minimum uncertainty states. Accordingly we assume that $\gamma$ is small and hence we neglect cube and higher powers of $\gamma$ to finally get

$$
Q\left(f_{\alpha}\right)=\frac{1}{4 \pi}\left(1-\frac{\gamma^{2}|\alpha|^{4}}{2}\right) \exp \left(-|\alpha|^{2} t^{2}-2|\alpha|^{3} t \gamma-|\alpha|^{4} \gamma^{2}+|\alpha|^{6} t^{2} \gamma^{2}\right)
$$

where $t=\theta_{2}-\theta_{1}-\frac{\gamma}{2}$. In Fig. 1, we have plotted $Q\left(f_{\alpha}\right)$ taking $\gamma=0.01$ and $t=1,2,3$ when $\lambda_{n}=n$ !, given from (20).

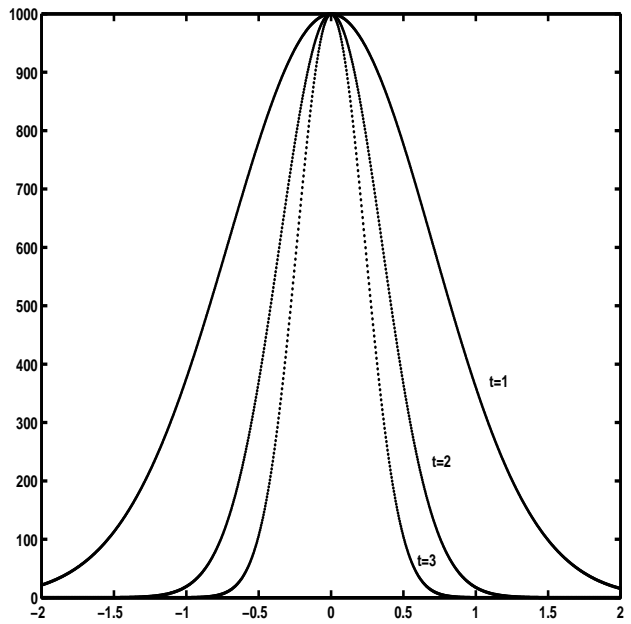

Fig. 1. Plot of $Q$ distribution as a function of $|\alpha|$

3.3. Squeezed state. For squeezed state [4]

$$
|\psi\rangle=c_{0} \sum_{n=0}^{\infty} \beta^{n} \frac{\lambda_{1} \lambda_{3} \ldots \lambda_{2 n-1}}{\lambda_{2} \lambda_{4} \ldots \lambda_{2 n}}|2 n\rangle
$$


we have

$$
\begin{aligned}
Q\left(f_{\alpha}\right) & =\frac{1}{\pi}\left|\left\langle f_{\alpha} \mid \psi\right\rangle\right|^{2} \\
& =\frac{1}{\pi}\left|\left\{\psi\left(|\alpha|^{2}\right)\right\}^{-1 / 2} \sum_{m=0}^{\infty} \frac{|\alpha|^{m}}{\lambda_{m}} e^{-i m \theta_{1}}\left\langle m\left|c_{0} \sum_{n=0}^{\infty} \beta^{n} \frac{\lambda_{1} \lambda_{3} \ldots \lambda_{2 n-1}}{\lambda_{2} \lambda_{4} \ldots \lambda_{2 n}}\right| 2 n\right\rangle\right|^{2} \\
& =\frac{1}{\pi}\left|\left\{\psi\left(|\alpha|^{2}\right)\right\}^{-1 / 2} c_{0} \sum_{m, n=0}^{\infty} \frac{|\alpha|^{m}}{\lambda_{m}} e^{-i m \theta_{1}} \beta^{n} \frac{\lambda_{1} \lambda_{3} \ldots \lambda_{2 n-1}}{\lambda_{2} \lambda_{4} \ldots \lambda_{2 n}}\langle m \mid 2 n\rangle\right|^{2} \\
& =\frac{1}{\pi}\left|\left\{\psi\left(|\alpha|^{2}\right)\right\}^{-1 / 2} c_{0} \sum_{n=0}^{\infty} \frac{|\alpha|^{2 n}}{\lambda_{2 n}} e^{-2 i n \theta_{1}} \beta^{n} \frac{\lambda_{1} \lambda_{3} \ldots \lambda_{2 n-1}}{\lambda_{2} \lambda_{4} \ldots \lambda_{2 n}} \lambda_{2 n}\right|^{2}
\end{aligned}
$$

For $\lambda_{n} \sim n$ ! we get

$$
\begin{aligned}
Q\left(f_{\alpha}\right) & \left.\left.\sim \frac{1}{\pi}\left|e^{-|\alpha|^{2} / 2} c_{0} \sum_{n=0}^{\infty} \frac{|\alpha|^{2 n}}{2^{n} n !} e^{-2 i n \theta_{1}}\right| \beta\right|^{n} e^{i n \theta_{2}}\right|^{2} \\
& =\frac{1}{\pi}\left|e^{-|\alpha|^{2} / 2} c_{0} \sum_{n=0}^{\infty} \frac{\left[|\alpha|^{2}|\beta| e^{\left.i\left(\theta_{2}-2 \theta_{1}\right) / 2\right]^{n}}\right.}{n !}\right|^{2} \\
& =\frac{1}{\pi}\left|e^{-|\alpha|^{2} / 2} c_{0} e^{\left[|\alpha|^{2}|\beta| e^{i\left(\theta_{2}-2 \theta_{1}\right)} / 2\right]}\right|^{2} \\
& =\frac{\left|c_{0}\right|^{2}}{\pi} e^{-|\alpha|^{2}} e^{|\alpha|^{2}|\beta| \cos \left(\theta_{2}-2 \theta_{1}\right)} \\
& =\frac{\left|c_{0}\right|^{2}}{\pi} e^{-|\alpha|^{2}\left[1-|\beta| \cos \left(\theta_{2}-2 \theta_{1}\right)\right]}
\end{aligned}
$$

In Fig. 2, we have plotted $Q\left(f_{\alpha}\right)$ when $\lambda_{n}=n$ !, obtained from (23).

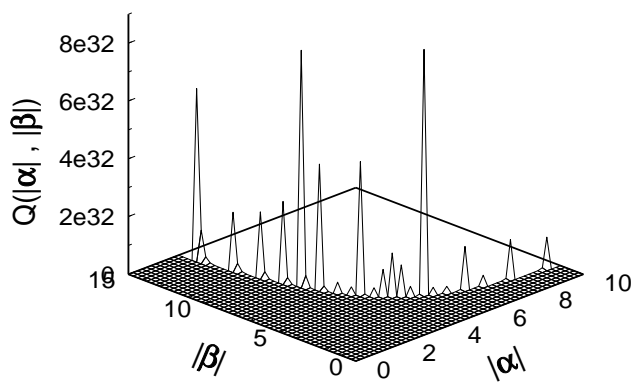

Fig. 2. Plot of $Q$ distribution as a function of $|\alpha|$ and $|\beta|$

3.4. $k$-Photon coherent state. Let us try to obtain the eigenstate of the operator $a^{k}$ where $a$ is the annihilation operator of the field,

$$
a^{k}\left|\alpha_{k}\right\rangle=\alpha_{k}\left|\alpha_{k}\right\rangle
$$

where

$$
\left|\alpha_{k}\right\rangle=\sum_{n=0}^{\infty} c_{n}^{(k)}|n\rangle .
$$


Then

$$
\begin{aligned}
a^{k}\left|\alpha_{k}\right\rangle & =\sum_{n=0}^{\infty} c_{n}^{(k)} a^{k}|n\rangle \\
& =\sum_{n=1}^{\infty} c_{n}^{(k)} a^{k-1} \frac{\lambda_{n}}{\lambda_{n-1}}|n-1\rangle \\
& =\ldots \ldots \ldots \ldots \ldots \\
& =\sum_{n=k}^{\infty} c_{n}^{(k)} \frac{\lambda_{n}}{\lambda_{n-1}} \frac{\lambda_{n-1}}{\lambda_{n-2}} \ldots \frac{\lambda_{n-(k-1)}}{\lambda_{n-k}}|n-k\rangle \\
& =\sum_{n=k}^{\infty} c_{n}^{(k)} \frac{\lambda_{n}}{\lambda_{n-k}}|n-k\rangle \\
& =\sum_{n=0}^{\infty} c_{n+k}^{(k)} \frac{\lambda_{n+k}}{\lambda_{n}}|n\rangle .
\end{aligned}
$$

Again

$$
\alpha_{k}\left|\alpha_{k}\right\rangle=\sum_{n=0}^{\infty} \alpha_{k} c_{n}^{(k)}|n\rangle .
$$

From (24), (26) and (27) we now have

$$
c_{n+k}^{(k)} \frac{\lambda_{n+k}}{\lambda_{n}}=\alpha_{k} c_{n}^{(k)}
$$

which gives

$$
c_{n}^{(k)}=\frac{\lambda_{n-k}}{\lambda_{n}} \alpha_{k} c_{n-k}^{(k)} .
$$

In (29) we replace $n$ by $n-k$ to get

$$
\begin{aligned}
c_{n}^{(k)} & =\frac{\lambda_{n-k}}{\lambda_{n}} \alpha_{k} c_{n-k}^{(k)} \\
& =\frac{\lambda_{n-k}}{\lambda_{n}} \alpha_{k} \frac{\lambda_{n-2 k}}{\lambda_{n-k}} \alpha_{k} c_{n-2 k}^{(k)} \\
& =\ldots \cdots \cdots \cdots \cdots \cdots \cdots \\
& =\frac{\lambda_{n-k}}{\lambda_{n}} \alpha_{k} \frac{\lambda_{n-2 k}}{\lambda_{n-k}} \alpha_{k} \cdots \frac{\lambda_{n-n k}}{\lambda_{n-(n-1) k}} \alpha_{k} c_{n-n k}^{(k)} \\
& =\left(\alpha_{k}\right)^{n} \frac{\lambda_{n-n k}}{\lambda_{n}} c_{n-n k}^{(k)} .
\end{aligned}
$$

We now have

$$
\begin{aligned}
\left|\alpha_{k}\right\rangle & =\sum_{n=0}^{\infty} c_{n}^{(k)}|n\rangle \\
& =\sum_{n=0}^{\infty}\left(\alpha_{k}\right)^{n} \frac{\lambda_{n-n k}}{\lambda_{n}} c_{n-n k}^{(k)}|n\rangle
\end{aligned}
$$




$$
\begin{aligned}
& =\sum_{n=0}^{\infty}\left(\alpha_{k}\right)^{(n k+m)} \frac{\lambda_{m}}{\lambda_{n k+m}} c_{m}^{(k)}|n k+m\rangle \\
& =\sum_{n=0}^{\infty}\left(\alpha_{k}^{k}\right)^{n} \alpha_{k}^{m} \frac{\lambda_{m}}{\lambda_{n k+m}} c_{m}^{(k)}|n k+m\rangle \\
& =\alpha_{k}^{m} c_{m}^{(k)} \sum_{n=0}^{\infty}\left(\alpha_{k}^{k}\right)^{n} \frac{\lambda_{m}}{\lambda_{n k+m}}|n k+m\rangle \\
& =C_{m} \sum_{n=0}^{\infty}\left(\alpha_{k}\right)^{n} \frac{\lambda_{m}}{\lambda_{n k+m}}|n k+m\rangle .
\end{aligned}
$$

In (31) we have replaced $n-n k$ by $m$ in step 2 and in step 6 we have taken $C_{m} \equiv \alpha_{k}^{m} c_{m}^{(k)}$, $\alpha_{k}^{k} \equiv \alpha_{k}$.

By taking the trivial phase we find the normalization constant $C_{m}$ to be

$$
C_{m}=\left[\sum_{n=0}^{\infty}\left|\alpha_{k}\right|^{2 n} \frac{\lambda_{m}^{2}}{\lambda_{m+n k}}\right]^{-1 / 2}
$$

whenever the series is convergent. In particular, when $\lambda_{n} \sim n$ !, we actually see that the series is convergent.

Then, finally we get $k$-photon coherent state to be

$$
\left|\alpha_{k}\right\rangle=\left[\sum_{n=0}^{\infty}\left|\alpha_{k}\right|^{2 n} \frac{\lambda_{m}^{2}}{\lambda_{m+n k}}\right]^{-1 / 2} \sum_{n=0}^{\infty}\left(\alpha_{k}\right)^{n} \frac{\lambda_{m}}{\lambda_{m+n k}}|m+n k\rangle .
$$

To describe the quasi-probability distribution for $k$-photon coherent state we rewrite the state $\left|\alpha_{k}\right\rangle$ as

$$
\left|\alpha_{k}\right\rangle=C_{m} \sum \frac{\lambda_{m}}{\lambda_{n}}\left(\alpha_{k}\right)^{n}|n\rangle
$$

Now

$$
\begin{aligned}
Q\left(f_{\alpha}\right) & =\frac{1}{\pi}\left|\left\langle f_{\alpha} \mid \alpha_{k}\right\rangle\right|^{2} \\
& =\frac{1}{\pi}\left|\left\{\psi\left(|\alpha|^{2}\right)\right\}^{-1 / 2} \sum_{l=0}^{\infty} \frac{|\alpha|^{l}}{\lambda_{l}} e^{-i l \theta_{1}}\left\langle l\left|C_{m} \sum_{n=0}^{\infty} \frac{\lambda_{m}}{\lambda_{n}}\left(\alpha_{k}\right)^{n}\right| n\right\rangle\right|^{2} \\
& =\frac{1}{\pi}\left|\left\{\psi\left(|\alpha|^{2}\right)\right\}^{-1 / 2} \sum_{l, n=0}^{\infty} \frac{|\alpha|^{l}}{\lambda_{l}} e^{-i l \theta_{1}} C_{m} \frac{\lambda_{m}}{\lambda_{n}}\left(\alpha_{k}\right)^{n}\langle l \mid n\rangle\right|^{2} \\
& =\frac{1}{\pi}\left|\left\{\psi\left(|\alpha|^{2}\right)\right\}^{-1 / 2} \sum_{n=0}^{\infty} \frac{|\alpha|^{n}}{\lambda_{n}} e^{-i n \theta_{1}} C_{m} \frac{\lambda_{m}}{\lambda_{n}}\left(\alpha_{k}\right)^{n}\langle n \mid n\rangle\right|^{2} \\
& =\frac{1}{\pi}\left|\left\{\psi\left(|\alpha|^{2}\right)\right\}^{-1 / 2} \sum_{n=0}^{\infty} \frac{|\alpha|^{n}}{\lambda_{n}} e^{-i n \theta_{1}} C_{m} \frac{\lambda_{m}}{\lambda_{n}}\left(\alpha_{k}\right)^{n} \lambda_{n}\right|^{2}
\end{aligned}
$$




$$
\begin{aligned}
& =\left.\left.\frac{1}{\pi}\left|C_{m}\right|^{2}\left\{\psi\left(|\alpha|^{2}\right)\right\}^{-1}\left|\sum_{n=0}^{\infty}\right| \alpha\right|^{n} e^{-i n \theta_{1}} \frac{\lambda_{m}}{\lambda_{n}}\left(\alpha_{k}\right)^{n}\right|^{2} \\
& =\left.\left.\frac{1}{\pi}\left|C_{m}\right|^{2}\left\{\psi\left(|\alpha|^{2}\right)\right\}^{-1}\left|\sum_{n=0}^{\infty} \frac{\lambda_{m}}{\lambda_{n}}\right| \alpha\right|^{n} e^{-i n \theta_{1}}\left(\alpha_{k}\right)^{n}\right|^{2} \\
& =\left.\left.\frac{1}{\pi}\left|C_{m}\right|^{2}\left\{\psi\left(|\alpha|^{2}\right)\right\}^{-1}\left|\sum_{n=0}^{\infty} \frac{\lambda_{m}}{\lambda_{n}}\right| \alpha\right|^{n}\left|\alpha_{k}\right|^{n} e^{i n\left(\theta_{2}-\theta_{1}\right)}\right|^{2} .
\end{aligned}
$$

For $\lambda_{n} \sim n$ ! we get

$$
\begin{aligned}
Q\left(f_{\alpha}\right) & \left.\left.\sim \frac{1}{\pi} e^{-|\alpha|^{2}}(m !)^{-2} e^{-|\alpha|^{2}}(m !)^{2}\left|\sum_{n=0}^{\infty} \frac{1}{n !}\right| \alpha\right|^{n}\left|\alpha_{k}\right|^{n} e^{i n\left(\theta_{2}-\theta_{1}\right)}\right|^{2} \\
& =\frac{1}{\pi} e^{-2|\alpha|^{2}}\left|\sum_{n=0}^{\infty} \frac{\left(|\alpha|\left|\alpha \alpha_{k}\right| e^{i\left(\theta_{2}-\theta_{1}\right)}\right)^{n}}{n !}\right|^{2} \\
& =\frac{1}{\pi} e^{-2|\alpha|^{2}}\left|e^{\left(|\alpha|\left|\alpha_{k}\right| e^{i\left(\theta_{2}-\theta_{1}\right)}\right)}\right|^{2} \\
& =\frac{1}{\pi} e^{-2|\alpha|^{2}}\left|e^{\left(|\alpha|\left|\alpha_{k}\right| \cos \left(\theta_{2}-\theta_{1}\right)+i|\alpha|\left|\alpha_{k}\right| \sin \left(\theta_{2}-\theta_{1}\right)\right)}\right|^{2} \\
& =\frac{1}{\pi} e^{-2|\alpha|^{2}} e^{2|\alpha|\left|\alpha_{k}\right| \cos \left(\theta_{2}-\theta_{1}\right)} \\
& =\frac{1}{\pi} e^{-2|\alpha|\left\{|\alpha|-\left|\alpha_{k}\right| \cos \left(\theta_{2}-\theta_{1}\right)\right\}}
\end{aligned}
$$

From (36) we now have

$$
Q\left(f_{\alpha}\right)=\frac{1}{\pi} e^{-2|\alpha|\left\{|\alpha|-\left|\alpha_{k}\right| \cos \left(\theta_{2}-\theta_{1}\right)\right\}} .
$$

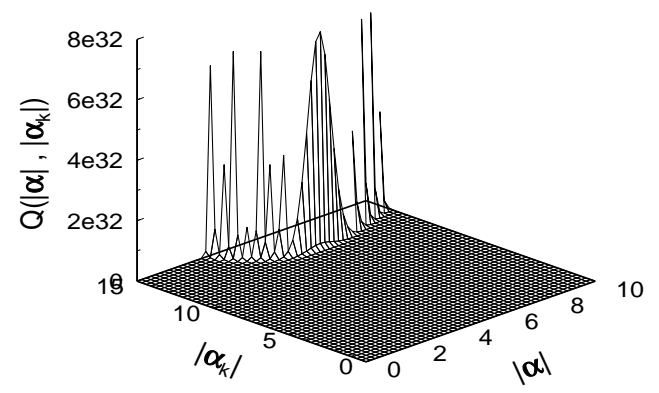

Fig. 3. Plot of $Q$ distribution as a function of $|\alpha|$ and $\left|\alpha_{k}\right|$

In Fig. 3, we have plotted $Q\left(f_{\alpha}\right)$ when $\lambda_{n}=n$ ! , obtained from (37).

4. Conclusion. Thus we have shown the usefulness of asymptotic approximation to study quasi-probability distribution function. This method helps us to express $Q$ functions by an analytic expression instead of cumbersome series expression and in its turn gives a better understanding of $Q$ function for incoherent states, Kerr states, squeezed states and $k$-photon coherent states in interacting Fock space. 
Acknowledgements. The authors wish to thank the referee for his extensive comments on the earlier version of the paper. The work of the second author is supported by a Council of Scientific and Industrial Research (India) fellowship.

\section{References}

[1] L. Accardi and W. Bożejko, Interacting Fock spaces and gaussianisation of probability measures, Infinite Dimensional Analysis, QP and Related Topics 1 (1998), 663-670.

[2] L. Accardi, Y. G. Lu and I. Volovich, Quantum Theory and its Stochastic Limit, Springer, 2002.

[3] J. Janszky, M. G. Kim and M. S. Kim, Quasi-probability and nonclassicality of fields, Phys. Rev. A 53 (1996), 502-507.

[4] P. K. Das, Coherent states and squeezed states in interacting Fock space, International Journal of Theoretical Physics 41 (2002).

[5] P. Meystre and M. Sargent III, Elements of Quantum Optics, Springer, 1999.

[6] M. O. Terra Cunha, Quasi-probability and probability distributions for spin $1 / 2$ states, quant-ph/0102038 v1 (2001).

[7] T. Opatrný, V. Bužek, J. Bajer and G. Drobný, Propensities in discrete phase spaces: $Q$ function of a state in a finite-dimensional Hilbert space, Phys. Rev. A 52 (1995), 2419-2428.

[8] C. A. Miller, J. Hilsenbeck and H. Risken, Asymptotic approximations for the Q function in the Jaynes-Cummings model, Phys. Rev. A 46 (1992), 4323.

[9] A. D. Wilson-Gordon, V. Bužek and P. L. Knight, Statistical and phase properties of displaced Kerr states, Phys. Rev. A 44 (1991), 7647. 